\title{
Regionale Muster im gesundheitlichen Wohlbefinden der Schweizer Bevölkerung

\author{
Untersuchung des gesundheitlichen Wohlbefindens aufgrund der Schweizerischen \\ Gesundheitsbefragung 1997 und der Schweizer Raumtypologien
}

\section{Charis Keller-Lengen, Zürich}

\section{Einleitung}

Der Wandel der natürlichen und soziokulturellen Umwelt stellt eine Herausforderung an unsere Gesellschaft dar. Neben Veränderungen in ökonomischen und ökologischen Bereichen haben der Wandel der Arbeitsbedingungen, der sozialen Beziehungen, aber auch der Lebensstile Auswirkungen auf die Gesundheit und das Wohlbefinden. Wie wichtig der sozioökonomische Status eines Individuums für eine gute oder schlechte Gesundheit ist, zeigen Studien von Miles (1991) und BartLey (1994). Sie stellten fest, dass Angehörige der unteren sozialen Klassen, Arbeitslose und Frauen im Verhältnis zu sozioökonomisch Bessergestellten häufiger von einer schlechteren Gesundheit berichten. Die Ursachen für die gesundheitlichen Unterschiede sind vermutlich komplexer als angenommen (Mitchell et al. 2000). Neben sozioökonomischen Faktoren wie Beruf, Bildung, Einkommen scheinen auch vom Individuum nicht direkt beeinflussbare kollektive Faktoren und Rahmenbedingungen eine Rolle für die Gesundheit und das Wohlbefinden zu spielen. Für geographische Ansätze sind gebietsgebundene Faktoren wie Charakteristika des Wohnortes und des sozialen Umfelds in der Nachbarschaft, das Vertrauen und die Interaktion mit der Gemeinschaft sowie die regionale Identität besonders erfolgversprechend (SUbRamanian et al. 2001; LOCHNER et al. 2000).

In der Schweiz werden seit einigen Jahren soziale und gesundheitliche Unterschiede in verschiedenen Regionen diskutiert. BopP und GUTZwILler setzten sich mit den regionalen Sterblichkeitsunterschieden in der Schweiz und Unterschieden in der Gesamtsterblichkeit zwischen Grossstadt und Umland (BOPP \& GuTZWILLER 1999) auseinander. Koller zeigt in seinem Bericht «Regionale Unterschiede und Gesundheit», basierend auf den Schweizerischen Gesundheitsbefragungen 1992/93 und 1997, dass Kultur und Geographie wichtige Grössen im Zusammenhang mit Gesundheit sind. So sind beispielsweise Unterschiede im gesundheitlichen und psychischen Wohlbefinden nach Höhenlage, in der Hospitalisationsdauer sowie in der Prävalenz individueller Risikofaktoren feststellbar (Koller 2000).
Aufgrund der kleinteiligen Vielfalt an Sprachen, Kulturen, Identitäten und sozialem Kapital sowie den dahinter stehenden Einstellungen und Weltbildern ist die Schweiz prädestiniert für die Analyse der Interaktion von individuellen und kollektiven Einflussfaktoren (compositional vs. contextual effects). Als kollektive Faktoren wurden bezüglich des gesundheitlichen Wohlbefindens verschiedene, vom Bundesamt für Statistik gebildete Raumindikatoren wie die Sprachgebiete (3 Kategorien), die sieben Grossregionen (7 Kategorien), der Indikator Stadt/Land (2 Kategorien), die Gebiete nach Gemeindegrösse (8 Kategorien), die 22 Gemeindetypen (22 Typen) und ihre Klassifizierung (8 Klassen) sowie die Gebiete in Höhe über Meer (4 Kategorien) untersucht. In diesem Beitrag soll das gesundheitliche Wohlbefinden anhand der von Joye, Schuler, Nef und BASSAND nach dem Zentrum-Peripherien-Ansatz entwickelten und seit den 1980er Jahren verwendeten Raumtypologien (22 Gemeindetypen und ihre Klassifizierung) in verschiedenen Schweizer Gebieten erläutert werden (JoYE et al.1988). Wie nun das gesundheitliche Wohlbefinden der Bevölkerung in diesen teils räumlich zusammenhängenden Regionen und teils nicht zusammenhängenden Raumtypen eingeschätzt wird, soll unter Berücksichtigung von Alter, Geschlecht, Bildung und sozioprofessionellem Status anhand der Schweizerischen Gesundheitsbefragung 1997 gezeigt werden. Als statistisches Verfahren wurde die einfache Korrespondenzanalyse gewählt. Dass die Erfassung des gesundheitlichen Wohlbefindens als wichtige Variable sinnvoll ist, zeigen diverse Studien (SUNDQUIST \& JoHANSSON 1997). Ein schlechtes gesundheitliches Wohlbefinden ist ein starker Prädiktor für die Mortalität.

\section{Methoden}

\subsection{Datengrundlage}

Als Datengrundlage dienten die Individualdaten der Schweizerischen Gesundheitsbefragung (SGB) 1997. Diese beruhte auf einer 2-stufigen, geschichteten Zufallsstichprobe mit 13.004 befragten Personen aus der Schweizer Wohnbevölkerung (Schweizerinnen und Schweizer, niedergelassene Ausländerinnen und Ausländer) von 15 und mehr Jahren, die in einem Privathaushalt mit Telefonanschluss lebten. Um die Repräsentativität der von der Stichprobe gewonnenen Aussagen für die Schweizer Wohnbevölkerung zu gewährleisten, wurde vom Bundesamt für Statistik ein Gewichtungsfaktor 
nach dem Horwitz-Thompson-Schätzer eingeführt. Für die Gesundheitsbefragung wurden Stichprobe und Gesamtbevölkerung in Bezug auf Geschlecht, Alter, Wohnort und Nationalität (Schweizer/Ausländer) verglichen. Die gewichteten Daten betrafen die Schweizer Wohnbevölkerung von 15 und mehr Jahren Mitte 1997, insgesamt 5.889.186 Personen (CALMONTE et al. 2000). In den Ergebnissen wurden die Analysen der gewichteten Daten interpretiert.

\subsection{Gesundheitliches Wohlbefinden}

Die subjektive Einschätzung des gesundheitlichen Wohlbefindens wurde in der SGB mit der Frage erfasst: «Wie geht es Ihnen zur Zeit gesundheitlich?», wobei darauf mit «sehr gut», «gut», «mittelmässig», «schlecht» und «sehr schlecht» geantwortet werden konnte. Da die Stichprobengrösse der Merkmalsausprägung «sehr schlecht» unter 3\% lag und dies in der Auswertung zu Verzerrungseffekten hätte führen können, wurden die Kategorien «schlecht» und «sehr schlecht» in der Korrespondenzanalyse zusammengefasst.

\subsection{Raumtypologien}

Folgende Raumtypen kamen zur Anwendung: die 22 Gemeindetypen und die 22 Gemeindetypen in 8 Klassen. Sie wurden aufgrund der Volkszählung 1990 gebildet und basierten auf dem Gemeinde Master File (GMF). Wichtige Grundlage war das Zentren-Peripherien-Modell nach Joye, Schuler, Nef und BasSAND (JoYE et al. 1988). Die Raumtypen wurden derart aufgebaut, dass zuerst nach Gross-, Mittel- und Kleinzentralen sowie peripheren Regionen unterschieden wurde. Innerhalb dieser wurden für die Agglomerationsgemeinden Kriterien wie Arbeitsplatzdichte, Wegpendleranteil, Gebäudestruktur und Einkommen der Bevölkerung bestimmend, für Gemeinden ausserhalb der Agglomerationen jedoch die dominante Wirtschaftsstruktur der Bevölkerung. Demographie und spezifisches Migrationsverhalten wurden in ein paar Sondertypen ebenfalls berücksichtigt (SCHULER \& JOYE 1997). Die Kategorien der Raumindikatoren sind aus der Tabelle 1 ersichtlich.

\subsection{Alter, Geschlecht, Bildung und sozioprofessio- nelle Kategorien}

Alter und Geschlecht konnten zu sechs Kategorien mit 15- bis 35-jährigen, 36- bis 65-jährigen und über 65-jährigen Männern und Frauen zusammengefasst werden (Tabelle 1). Das Bildungsniveau wurde aufgrund der Fragen bezüglich obligatorischer Schule, Ausbildung nach der obligatorischen Schule, Zweitausbildung und der höchsten abgeschlossenen Ausbildung entwickelt. Die sozioprofessionellen Kategorien wurden anhand von Fragen zur beruflichen Stellung, zur Anzahl Angestellten sowie zum zuletzt ausgeführten Beruf, ferner zu Arbeitnehmern und Selbständigen gebildet (Tabelle 1).

\subsection{Statistische Methoden}

Da es sich bei der Variablen «Gesundheitliches Wohlbefinden" und den Gebietsindikatoren um ordinalund nominalskalierte, d.h. kategoriale Daten handelt, wurden die Kontingenztabellen mit Chi-Quadrat-Statistik auf Unabhängigkeit und Homogenität getestet. Die Stärke des Zusammenhangs zwischen zwei kategorial aufgegliederten Merkmalen wurde mit CRAMERS $\mathrm{V}$ geprüft. Als geeignete Methode zur Untersuchung des kategorialen Datenmaterials wurde die in der Geographie selten zur Anwendung kommende Korrespondenzanalyse gewählt, da sie im Gegensatz zu den loglinearen Modellen, die nur eine eng begrenzte Anzahl der Variablen(ausprägungen) zulässt, eine nahezu unbegrenzte Anzahl der Variablen analysiert. Die Korrespondenzanalyse ist ein exploratives Verfahren zur graphischen und numerischen Darstellung von Zeilen und Spalten beliebiger Kontingenztabellen. Wie bei der multidimensionalen Skalierung ist die Distanzinterpretation zwischen Variablen und ebenso zwischen den Objekten von Bedeutung. Faktorenwerte der Objekte und Variablen können ähnlich wie bei der Hauptkomponentenanalyse bezüglich der einzelnen Achsen berechnet werden. Bei der Korrespondenzanalyse ist im Gegensatz zur Hauptkomponentenanalyse die graphische Darstellung von zentraler Bedeutung (BLasius 2001).

\section{Ergebnisse}

Im Schnitt fühlten sich $83 \%$ der Schweizer Bevölkerung gesundheitlich "gut» und «sehr gut». Die restlichen $17 \%$ fühlten sich «mittelmässig», «schlecht» und «sehr schlecht». Aufgrund dieser Frequenzen wird die statistische Problematik erkennbar. Die Profile «sehr gutes» und «gutes» Wohlbefinden sind aufgrund ihrer Stichprobengrösse aussagekräftiger als die Profile «mittelmässiges» und «schlechtes/sehr schlechtes» gesundheitliches Wohlbefinden. Da die relativen Häufigkeiten der 22 Gemeindetypen bezüglich des gesundheitlichen Wohlbefindens oft bei $3 \%$ liegen, sind die Ergebnisse bezüglich der 22 Gemeindetypologien mit Vorsicht zu geniessen.

Aufgrund des Korrelationsmasses von Cramer, das bei völliger Unabhängigkeit den Wert Null aufweist, scheint der Zusammenhang zwischen Alter/Geschlecht, Bildung und sozioprofessionellen Kategorien und gesundheitlichem Wohlbefinden stärker zu sein als zwischen den Raumtypen und dem gesundheitlichen Wohlbefinden. Obwohl der Zusammenhang zwischen Raumtypen und gesundheitlichem Wohlbefinden zwei- bis viermal schwächer ist, lohnt es sich, anhand der Korrespondenzanalyse nach allfälligen Mustern zu suchen. Auch im weissen Rauschen können Strukturen erscheinen, die sozialgeographisch interpretierbar sind. 


\begin{tabular}{|c|c|c|c|c|}
\hline \multirow[t]{2}{*}{$\begin{array}{l}\text { Raumtypen, Alter/Geschlecht, Bildung und } \\
\text { sozioprofessionelle Kategorien }\end{array}$} & \multicolumn{4}{|c|}{$\begin{array}{l}\text { Gesundheitliches Wohlbefinden in Pro- } \\
\text { zent der Gesamtstichprobe }\end{array}$} \\
\hline & $\begin{array}{l}\text { sehr } \\
\text { gut }\end{array}$ & gut & $\begin{array}{l}\text { mittel- } \\
\text { mässig }\end{array}$ & $\begin{array}{l}\text { schlecht/ } \\
\text { sehr schlecht }\end{array}$ \\
\hline \multicolumn{5}{|l|}{22 Gemeindetypen } \\
\hline \multicolumn{5}{|l|}{ Chi-Quadrat $\mathrm{p}=<0,0001$, Cramers $\mathrm{V}=0,05$} \\
\hline GT1 = Grosszentren & 3,4 & 7,8 & 1,8 & 0,7 \\
\hline GT2 $=$ Mittelzentren & 2,6 & 6,1 & 1,6 & 0,6 \\
\hline GT3 = Kleinzentren & 2,0 & 4,1 & 0,8 & 0,3 \\
\hline GT4 = Peripheriezentren & 0,4 & 1,0 & 0,2 & 0,1 \\
\hline GT5 $=$ Reiche Gemeinden & 1,2 & 2,5 & 0,5 & 0,1 \\
\hline GT6 = Touristische Gemeinden & 0,6 & 1,0 & 0,2 & 0,1 \\
\hline GT7 = Semitouristische Gemeinden & 0,3 & 0,5 & 0,1 & 0,0 \\
\hline GT8 = Heim- und Anstaltsgemeinden & 0,1 & 0,4 & 0,0 & 0,0 \\
\hline GT9 = Arbeitsplatzgemeinden grosszentraler Regionen & 1,9 & 3,8 & 0,8 & 0,2 \\
\hline GT10 = Suburbane Wohngemeinden grosszentraler Regionen & 2,0 & 4,5 & 1,2 & 0,3 \\
\hline GT11 = Periurbane Gemeinden grosszentraler Regionen & 1,1 & 1,9 & 0,3 & 0,1 \\
\hline GT12 = Arbeitsplatzgemeinden nicht-grosszentraler Regionen & 1,8 & 4,0 & 1,0 & 0,3 \\
\hline GT13 = Suburbane Wohngemeinden nicht-grosszentraler Regionen & 1,2 & 2,3 & 0,4 & 0,2 \\
\hline GT14 = Periurbane Gemeinden nicht-grosszentraler Regionen & 2,1 & 4,3 & 1,0 & 0,2 \\
\hline GT15 = Zuzügergemeinden mit mässigem Wegpendleranteil & 1,1 & 2,1 & 0,5 & 0,2 \\
\hline GT16 = Einheimischengemeinden mit mässigem oder hohem Wegpendleranteil & 0,5 & 1,0 & 0,3 & 0,0 \\
\hline GT17 = Gemeinden mit industriell-tertiärer Erwerbsbevölkerung & 1,2 & 3,2 & 0,7 & 0,2 \\
\hline GT18 = Gemeinden mit industrieller Erwerbsbevölkerung & 1,0 & 2,2 & 0,4 & 0,1 \\
\hline GT19 = Gemeinden mit agrar-industrieller Erwerbsbevölkerung & 0,8 & 2,0 & 0,5 & 0,1 \\
\hline GT20 = Gemeinden mit agrar-tertiärer Erwerbsbevölkerung & 0,7 & 1,6 & 0,3 & 0,1 \\
\hline GT21 = Gemeinden mit agrarischer Erwerbsbevölkerung & 0,1 & 0,7 & 0,1 & 0,0 \\
\hline GT22 = Gemeinden mit starkem Bevölkerungsrückgang & 0,1 & 0,2 & 0,1 & 0,0 \\
\hline \multicolumn{5}{|l|}{22 Gemeindetypen in 8 Klassen } \\
\hline \multicolumn{5}{|l|}{ Chi-Quadrat $\mathrm{p}=<0,0001$, Cramers $\mathrm{V}=0,04$} \\
\hline GTKL1 = Zentren & 8,0 & 18,0 & 4,3 & 1,5 \\
\hline GTKL2 = Suburbane Gemeinden & 6,9 & 14,6 & 3,5 & 1,0 \\
\hline GTKL3 = Reiche Gemeinden & 1,2 & 2,5 & 0,5 & 0,1 \\
\hline GTKL4 = Periurbane Gemeinden & 3,2 & 6,1 & 1,3 & 0,3 \\
\hline GTKL5 = Touristische Gemeinden & 0,9 & 1,5 & 0,3 & 0,1 \\
\hline GTKL6 = Industriell-tertiäre Gemeinden & 2,8 & 6,8 & 1,3 & 0,4 \\
\hline GTKL7 = Ländliche Pendlergemeinden & 1,6 & 3,1 & 0,8 & 0,2 \\
\hline GTKL8 = Agrar-gemischte und periphere Gemeinden & 1,7 & 4,4 & 1,0 & 0,3 \\
\hline \multicolumn{5}{|l|}{ Alter und Geschlecht } \\
\hline \multicolumn{5}{|l|}{ Chi-Quadrat $\mathrm{p}=<0,0001$, Cramers $\mathrm{V}=0,13$} \\
\hline AS1 $=15$ - bis 35 -jährige Männer & 6,0 & 10,0 & 1,2 & 0,3 \\
\hline AS2 $=15$ - bis 35 -jährige Frauen & 5,4 & 10,2 & 1,5 & 0,4 \\
\hline AS3 $=36$ - bis 65 -jährige Männer & 6,4 & 13,9 & 2,5 & 0,9 \\
\hline AS4 $=36$ - bis 65 -jährige Frauen & 5,7 & 13,7 & 3,5 & 1,2 \\
\hline AS5 $=>65$-jährige Männer & 1,4 & 3,9 & 1,3 & 0,4 \\
\hline AS6 $=>65$-jährige Frauen & 1,5 & 5,3 & 2,9 & 0,8 \\
\hline \multicolumn{5}{|l|}{ Bildung } \\
\hline \multicolumn{5}{|l|}{ Chi-Quadrat $p=<0,0001$, Cramers $V=0,09$} \\
\hline B0 $=$ Ohne Ausbildung, unbestimmt & 0,9 & 1,7 & 0,3 & 0,2 \\
\hline B1 = Obligatorische Schule & 4,6 & 12,1 & 4,5 & 1,5 \\
\hline B2 $=$ Sekundarstufe & 15,5 & 33,4 & 6,8 & 2,0 \\
\hline B3 = Tertiärstufe & 5,3 & 9,7 & 1,3 & 0,3 \\
\hline \multicolumn{5}{|l|}{ Sozioprofessionelle Kategorien } \\
\hline \multicolumn{5}{|l|}{ Chi-Quadrat $\mathrm{p}=<0,0001$, CRAmERs $\mathrm{V}=0,06$} \\
\hline SPK1 = Höhere Führungskräfte, freie Berufe & 2,6 & 4,7 & 0.7 & 0,2 \\
\hline SPK2 = Höherqualifizierte nicht-manuelle Berufe, mittleres Kader & 7,5 & 15,0 & 2,8 & 0,7 \\
\hline SPK3 = Büroangestellte und andere nicht-manuelle Berufe & 7,1 & 15,4 & 3,4 & 1,0 \\
\hline SPK4 = Kleinunternehmer, selbständige Handwerker & 2,4 & 4,8 & 1,0 & 0,2 \\
\hline SPK5 = Vorarbeiter, qualifizierte manuelle Berufe & 3,8 & 9,4 & 2,3 & 0,8 \\
\hline SPK6 = An- und ungelernte manuelle Berufe & 3,2 & 7,8 & 2,4 & 0,9 \\
\hline Landesdurchschnitt & 26,27 & 56,9 & 12,8 & 4,0 \\
\hline
\end{tabular}

Tab. 1: Gesundheitliches Wohlbefinden, nach Raumtypologien, Alter/Geschlecht, Bildung und sozioprofessionellen Kategorien State of health according to spatial typology, age/gender, level of education and socio-professional categories Le bien-être physique, selon les typologies spatiales, l'agelle sexe, le niveau de formation et les catégories socio-professionnelles 


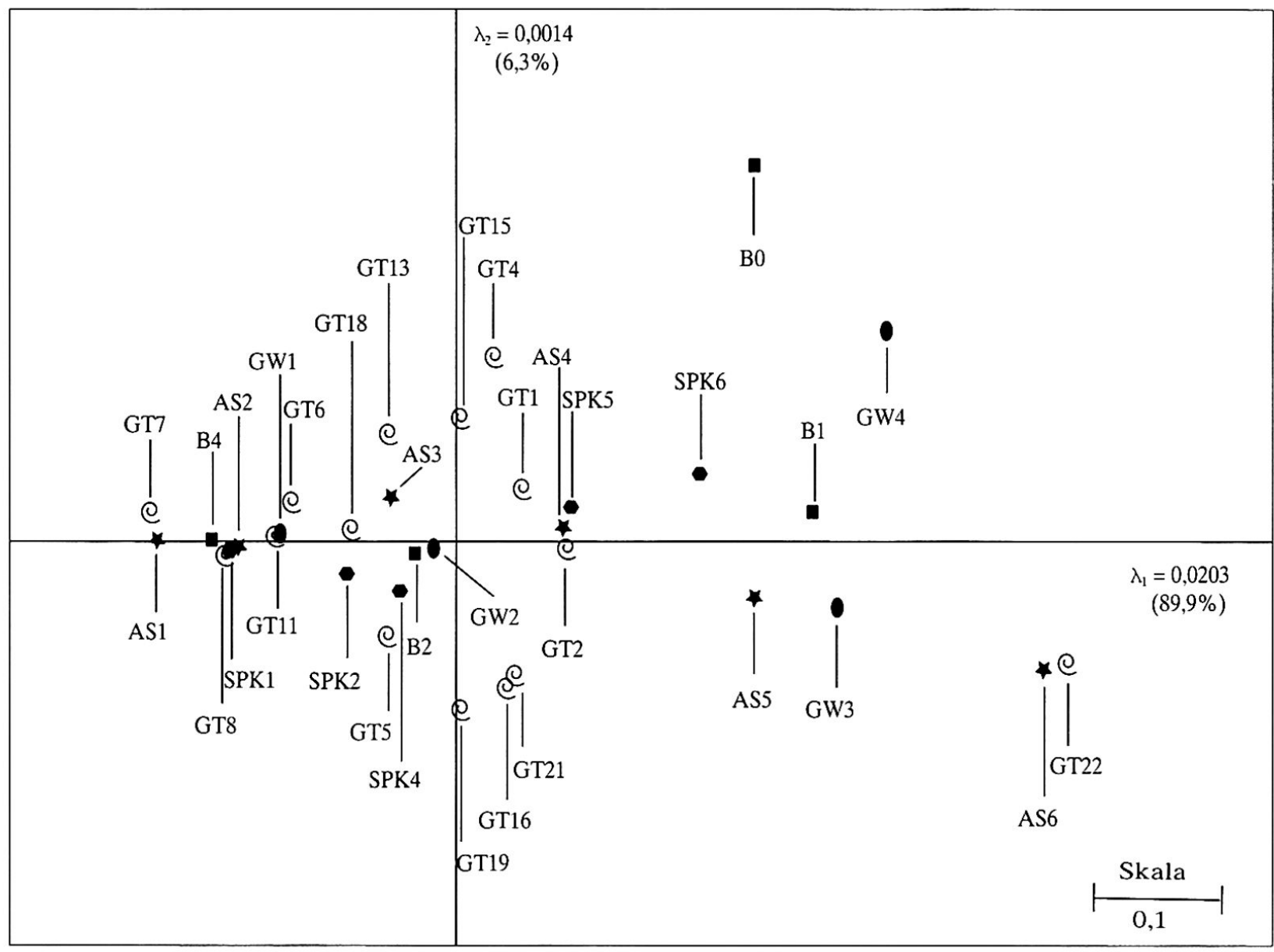

Abb. 1: Symmetrische Darstellung der Zeilen- und Spaltenprofile bezüglich gesundheitlichem Wohlbefinden und den 22 Gemeindetypen (Legende siehe Tabelle)

Symmetrical representation of bar and column profiles reflecting states of health in 22 communities (see table for legend)

Représentation symétrique des profils des lignes et des rubriques en fonction du bien-être physique et des 22 types de communes (Légende: voir le tableau)

Gesundheitliches Wohlbefinden und Gebiete nach Gemeindetypologien. Wenn das gesundheitliche Wohlbefinden bezüglich der Raumtypen nach dem ZentrumPeripherien-Ansatz untersucht wird und dabei die 22 Gemeindetypologien als Raummerkmal dienen, erhält man unter Berücksichtigung der soziodemographischen sowie sozioökonomischen Variablen eine Matrix von 38 Zeilen und 4 Spalten. Die Dimensionalität des Projektionsraumes ist somit 37. Die dazugehörigen Achsen werden durch Trägheitsgewichte (Inertia = quadrierte kanonische Korrelationskoeffizienten, Werte zwischen 0 und 1) in absteigender Ordnung und die dazugehörigen erklärenden Varianzen beschrieben. Die Inertia der ersten Achse ist $\lambda_{1}=0,0203$. Wird dieser Wert auf die Gesamtvariation der zu untersuchenden Daten, also auf die Gesamtinertia bezogen, so ist das Ergebnis die erklärende Varianz der ersten Achse. Erstaunlicherweise wird die Gesamtvarianz durch drei Achsen erklärt. Die erste Achse weist $89,9 \%$, die zweite $6,3 \%$ und die dritte $3,7 \%$ der erklärenden Varianz auf. In der geometrischen Darstellung (Abbildung 1) stehen somit die ersten zwei Dimensionen für 96,2\% der Gesamtvarianz. Werden die Ergebnisse inhaltlich interpretiert, so spiegelt die erste Achse in ordinaler Ordnung von links nach rechts das «sehr gute» (GW1) bis «schlechte/ sehr schlechte» (GW4) gesundheitliche Wohlbefinden sowie das Alter von 15 (AS1 und AS2) bis über 65 Jahre (AS5 und AS6) wider. Auch die Bildung lädt mit der Tertiärstufe bis zur obligatorischen Schule unter ordinaler Folge von links nach rechts auf die erste Achse. Nur die Gruppe ohne Ausbildung (B0) ist hierin eine Ausnahme. In derselben Weise laden auch die sozioprofessionellen Kategorien mit den höheren Führungskräften (SPK1) von links bis mit den an- und ungelernten manuellen Berufen (SPK6) nach rechts (Ausnahme SPK3) auf die erste Achse. Die linke Seite spiegelt 
somit die jungen, sich gesundheitlich wohl fühlenden, gebildeten und beruflich höher qualifizierten Frauen und Männer wider, im Gegensatz zur rechten Seite, die für eine ältere, gesundheitlich sich eher «schlecht» fühlende, weniger gebildete und in einfachen manuellen Berufen tätige Bevölkerung steht. Die zweite Achse, die nur $6,3 \%$ der verbleibenden Variation erklärt, könnte in Ansätzen im negativen Bereich durch die Peripherie (GT19, GT16, GT21) im Positiven durch die Zentren und die umliegenden Gemeinden (GT1, GT4, GT15) beschrieben werden. Mit «sehr gutem» gesundheitlichem Wohlbefinden sind in erster Linie die Profile der touristischen (GT6) und semitouristischen Gemeinden (GT7), die periurbanen Gemeinden grosszentraler Regionen (GT11) sowie Heim- und Anstaltsgemeinden (GT8) assoziiert. Auch im linken Achsenbereich zwischen «gutem» und "sehr gutem» gesundheitlichem Wohlbefinden stehen die Gemeinden mit industrieller Erwerbsbevölkerung (GT18), die reichen Gemeinden (GT5) und die suburbanen Wohngemeinden nicht grosszentraler Regionen (GT13). Mit «mässigem» und «schlechtem/sehr schlechtem» gesundheitlichem Wohlbefinden fallen besonders die Gemeinden mit starkem Bevölkerungsrückgang (GT22) auf. Interessanterweise sind sie sehr stark mit den über 65-jährigen Frauen assoziiert. Die Grosszentren (GT1), die Mittelzentren (GT2), die Peripheriezentren (GT4), die Zuzügergemeinden mit mässigem Wegpendleranteil (GT15), die Gemeinden der vorwiegend Einheimischen mit mässigem oder hohem Wegpendleranteil (GT16) und die Gemeinden mit agrarischer Erwerbsbevölkerung (GT21) kommen zwar auf die rechte Seite der ersten Achse zu liegen, sind aber doch noch mehr mit dem "guten" als dem «mittelmässigen» gesundheitlichen Wohlbefinden assoziiert. Ein «mittelmässiges» und «schlechtes/sehr schlechtes" gesundheitliches Wohlbefinden ist eher mit dem höheren Alter, einer tieferen Bildung und beruflichen Stellung assoziiert als mit den Raumtypen (ausser GT22). Zudem müssen die Resultate hier bezüglich der 22 Gemeindetypen wegen der geringen relativen Häufigkeit in jeder Zelle der zugrunde liegenden Kontingenztabelle mit Vorsicht interpretiert werden. Die Profile GT3, 9, 10, 12, 14, 17, 20, SPK3 und B3 kamen nahe beim Zentroid zu liegen und wurden nicht interpretiert.

Nach der Zusammenfassung der 22 Gemeindetypen in 8 Klassen verändert sich das vorher gezeichnete Bild wenig. Wieder erklären drei Dimensionen die Gesamtvarianz der Daten (Dimension $1=92,3 \%$, Dimension 2 $=4,8 \%$, Dimension $3=2,9 \%$ ). Die Achsen können wie oben beschrieben interpretiert werden. Die erste Achse links wird durch junge, gebildete, beruflich gut gestellte Männer und Frauen mit einem «guten» bis «sehr guten" gesundheitlichen Wohlbefinden bestimmt. Die rechte Seite zeichnet das Gegenteil. Die zweite Achse spiegelt oben eher die Zentren (GTKL1) mit ihrer Sub- urbia (GTKL2, GTKL7) und die industriell-tertiären Gemeinden wider, während unten die Peripherie (GTKL8) zu liegen kommt. Mit «sehr gutem» gesundheitlichem Wohlbefinden sind touristische Gemeinden (GTKL5) assoziiert. Zwischen «sehr gutem" und «gutem» gesundheitlichem Wohlbefinden stehen die periurbanen und reichen Gemeinden (GTKL4, GTKL3) (Abbildung 2).

\section{Diskussion}

Was ist nun für das gesundheitliche Wohlbefinden der Schweizer und Schweizerinnen wichtiger: sind es kollektive Einflussfaktoren wie die untersuchten Raumtypen oder die soziodemographischen und -ökonomischen Kriterien? Die Kontingenzkoeffizienten zeigen einen zwei- bis viermal höheren Zusammenhang zwischen gesundheitlichem Wohlbefinden und dem Merkmal Alter/Geschlecht (Cramers V $=0,13$ ) als zwischen gesundheitlichem Wohlbefinden und den untersuchten Raumtypen (CRAMERS V $=0,4$ und 0,5, Tabelle 1). Die Bildung (Cramers $V=0,09$ ) und die sozioprofessionellen Kategorien (Cramers V =0,06) weisen einen etwas stärkeren Zusammenhang auf als die Raumtypen. Es lohnt sich trotzdem nach allfälligen Strukturen und Mustern zu suchen und aufgrund der aus der Korrespondenzanalyse gewonnenen geometrischen Darstellung eine vorsichtige Interpretation bezüglich der Raumtypen zu wagen.

\section{1 «Gutes» und «sehr gutes» gesundheitliches Wohlbefinden}

Die periurbanen Gemeinden grosszentraler Regionen sind mit "sehr gutem" gesundheitlichem Wohlbefinden assoziiert und gehören meist dem äusseren Agglomerationsgürtel an. Der Anteil an Wohnungen in Ein- und Zweifamilienhäusern (jüngeren Datums, ältere Einfamilienhausgemeinden finden sich meist im Typ der reichen Gemeinden) beträgt mehr als $35 \%$ bis $40 \%$. Auch die Einkommen sind hier relativ hoch. Die Bevölkerung ist vergleichsweise jung und zeigt extrem hohe Wegpendleranteile. Der Landwirtschaftsanteil liegt leicht über dem nationalen Durchschnitt (JoYE et al. 1988). Die Ergebnisse bezüglich Alter/ Geschlecht, Bildung und sozioprofessionellen Kategorien bestätigen dieses Bild.

Die reichen Gemeinden sind über das Steueraufkommen, das Mietzinsniveau und den Direktorenanteil definiert. Gut zwei Drittel der Gemeinden dieses Typs gehören dem Umland der fünf Grosszentren an, vor allem von Zürich (Seegemeinden) und Genf. Dieser Typ zeigt nicht nur in seiner Definition, sondern auch bezüglich des «guten» bis «sehr guten» gesundheitlichen Wohlbefindens Affinitäten zum Typ periurbaner Gemeinden grosszentraler Regionen. 


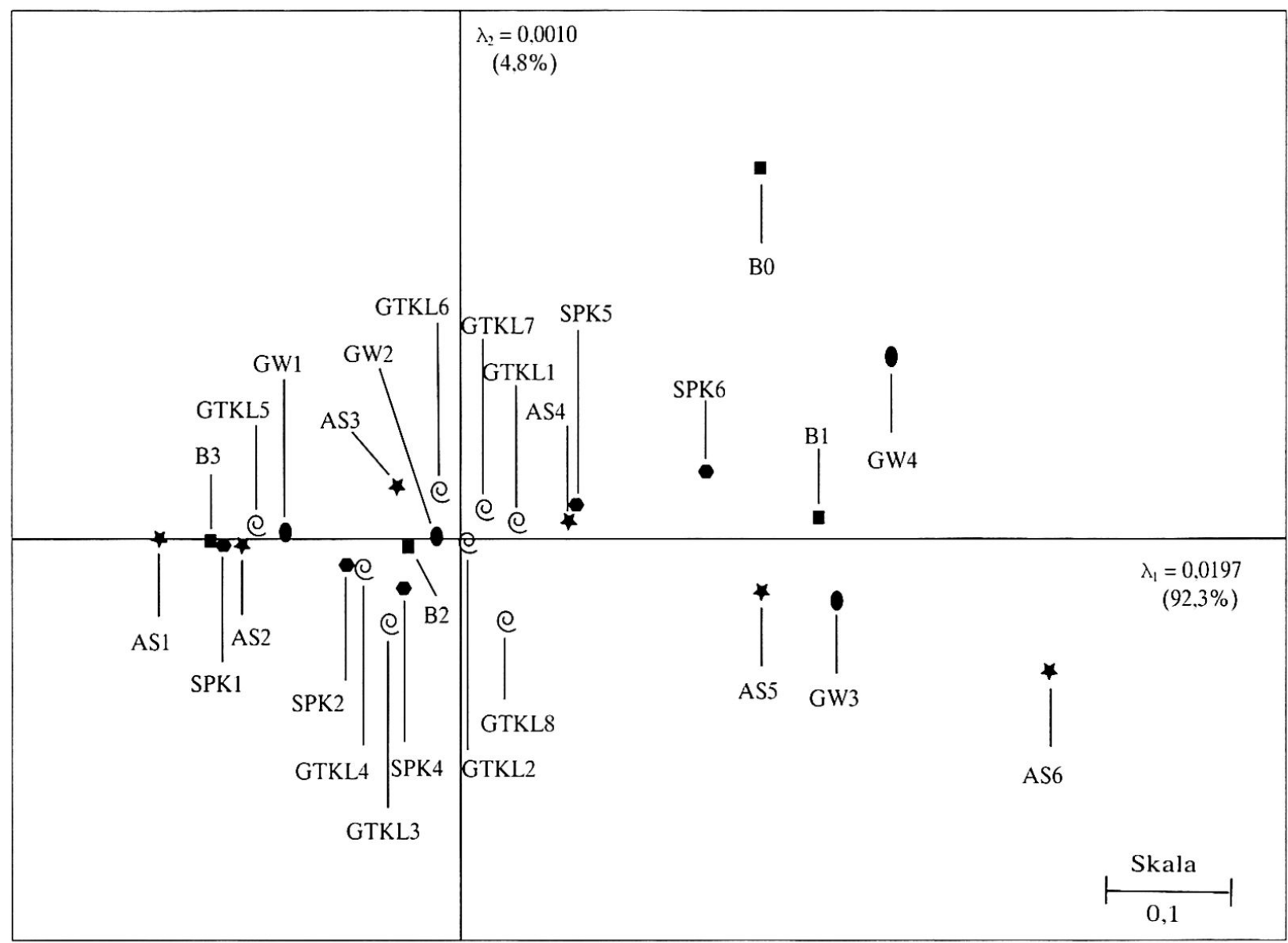

Abb. 2: Darstellung der Zeilen- und Spaltenprofile bezüglich gesundheitlichem Wohlbefinden und den Gemeindetypen in 8 Klassen (Legende siehe Tabelle)

Representation of bar and column profiles reflecting states of health in 22 communities according to 8 categories (see table for legend)

Représentation des profils de lignes et de rubriques selon le bien-être physique et les types de communes regroupées en 8 classes (Légende: voir le tableau)

Für die bezüglich des «sehr guten» gesundheitlichen Wohlbefindens auffälligen touristischen und semitouristischen Gemeinden sind Variablen wie Logiernächte, Zweitwohnungen und Erwerbstätige im Gastgewerbe massgebend. Dem touristischen Typ wurden grössere, klassische und auch jüngere Gemeinden zugewiesen. Sie liegen in den Kantonen Graubünden und Wallis, im Berner Oberland und am Vierwaldstättersee. Einzelne Bäderorte wurden ebenfalls berücksichtigt. Das Tessin ist nur mit wenigen Gemeinden vertreten. Lugano und Locarno sind als Zentren definiert. Im semitouristischen Gemeindetyp sind kleinere touristische Gemeinden wie Hasliberg, Lungern, Elm, Amden, Obersaxen, Cadenmario, Evolène im Alpenraum und Tessin sowie Vororte hochtouristischer Gemeinden wie Ringgenberg, Bever, Parpan, Brione sopra Minusio, Veytaux, Täsch oder Saas Almagell zusammengefasst. Die ländlichen Merkmale wie hoher Anteil der Landwirt- schaft, der Einheimischen, der Selbständigerwerbenden und der Wohneigentümer fallen hier ins Gewicht. Wegen der geringen Anzahl n muss dieses Resultat jedoch mit Vorsicht genossen werden. Die periurbanen, reichen und touristischen Gemeinden sind in den 8 Klassen ebenfalls stark mit «gutem» bis «sehr gutem» gesundheitlichem Wohlbefinden assoziiert.

\section{2 «Mittelmässiges» und «schlechtes» gesundheitli- ches Wohlbefinden}

Die Grosszentren liegen zwischen den Profilen «gutes» und «mittelmässiges» gesundheitliches Wohlbefinden. Im Vergleich zu den anderen Gemeindetypen geben die in Grosszentren lebenden Personen ein weniger gutes gesundheitliches Wohlbefinden an. Was die Grosszentren besonders auszeichnet, ist der hohe Zentralitätsgrad sowie eine besondere Bevölkerungsund Wohnstruktur. Hier sind der Kinderanteil, die 
Haushaltgrösse und der Anteil an Eigentumswohnungen am geringsten. Die höchste Bevölkerungsund Arbeitsplatzdichte sowie ein sehr hoher Ausländeranteil (besonders in Genf) sind für die Grosszentren charakteristisch. Die Mittelzentren der Gemeindetypologie, die 20 Städte mit $25^{\prime} 000$ bis $100^{\prime} 000$ Einwohnern auf sich vereinen, sind Zentren von mittelzentralen Regionen gemäss der Raumtypologie und von mittelgrossen Agglomerationen (z.B. Solothurn). Ihr Profil tendiert im Vergleich zu den Grosszentren noch mehr zum «mittelmässigen» gesundheitlichen Wohlbefinden.

Eine ähnliche Tendenz zum «mittelmässigen» Wohlbefinden, nur im peripheren Raum, zeigen die Gemeinden mit agrarischer Erwerbsbevölkerung, in denen mehr als $40 \%$ der Erwerbstätigen im Primärsektor beschäftigt sind. Da in diesem Gemeindetyp nur etwas mehr als $2 \%$ der Schweizer Wohnbevölkerung wohnen und auch der Anteil der mit «sehr schlecht» Antwortenden gesamtschweizerisch relativ gering ist, ist dieses Resultat mit Vorsicht zu bewerten. Die agrarischen Gemeinden sind in der Regel kleine geschlossene Dörfer und zum Teil auch grössere Einheiten in typischen Streusiedlungsgebieten des Alpenvorlandes (Napfgebiet, Schwarzenburg). Dieser Typ ist in der westlichen Landeshälfte mehr vertreten als in der östlichen. Der höchste Anteil an Selbständigerwerbenden, der geringste Anteil an Ausländern, Frauen und Geschiedenen, das tiefe Einkommen und die tiefen Mietpreise sind für diese in jeder Hinsicht sehr ländlichen Gemeinden typisch (Joye et al. 1988). Das Profil der agrar-gemischten und peripheren Gemeinden der Gemeindetypen nach 8 Klassen liegt beinahe am selben Ort im Projektionsraum und bestätigt mit einer höheren Anzahl $n$ diese Tendenz.

Gemeinden mit starkem Bevölkerungsrückgang, die mit «mässigem» und «schlechtem» gesundheitlichem Wohlbefinden assoziiert sind, stehen für eine extrem periphere Situation. In diesem Typ erscheinen Zwerggemeinden mit starkem Bevölkerungsverlust und grosser Überalterung. Dies zeigt sich vor allem in den Kantonen Tessin, Graubünden und Freiburg. Daher ist auch verständlich, dass das Profil GT22 so stark mit den über 65-jährigen Frauen assoziiert ist. Auch wenn die relative Häufigkeit bei diesem Typ gering ist, zeigt gerade dieses Ergebnis die Bedeutung des in dieser Studie verwendeten Korrespondenzanalysemodells.

\section{Fazit}

Aus den Untersuchungen geht klar hervor, dass besonders das Alter ein starker Prädiktor für das gesundheitliche Wohlbefinden ist. Auch der sozioökonomische Status scheint relevanter als die untersuchten Raumtypen zu sein. Dass Zentrum und Peripherie in den Raumtypen eher Assoziationen zum «mittelmässigen» gesundheitlichen Wohlbefinden aufweisen, ist interessant. Die beiden Extreme Zentrum und ländliche Peripherie weisen beide einen reduzierten sozialen Kontakt in unterschiedlicher Form und eine ältere, sich wenig reproduzierende Gesellschaft auf. Sie sind jedoch extrem gegensätzlich in den Merkmalen wie Einwohnerzahl und Ausländeranteil sowie in den Wirtschaftsstrukturen. Die Extreme und mit ihnen die erschwerten sozialen Kontakte scheinen das gesundheitliche Wohlbefinden zu belasten. Die in vielen Studien nachgewiesene Korrelation zwischen Sozioökonomie und Gesundheit (Wilkinson 1996; Miles 1991; Bartley 1994) kann mit dieser Studie bestätigt werden. Entsprechend sind auch die Gemeindetypen mit einer eher jungen, einkommensstarken Bevölkerung im Projektionsraum der gebildeten und beruflich gut positionierten Bevölkerung zu finden. Dass neben Beruf, Bildung und Einkommen auch vom Individuum nicht direkt beeinflussbare kollektive Faktoren und Rahmenbedingungen eine wichtige Rolle für die Gesundheit und das Wohlbefinden spielen können, kann mit diesen Ergebnissen nicht von der Hand gewiesen werden. Dass sie aber wichtiger als das Alter und der sozioökonomische Status sind, konnte hier nicht gezeigt werden.

\section{Dank}

Für die finanzielle Unterstützung des Projekts «Gesundheitslandschaften der Schweiz» sei dem Forschungskredit der Universität Zürich und dem Programm Marie Heim-Vögtlin, Schweizerischer Nationalfonds, gedankt.

\section{Literatur}

BARTLEY, M. (1994): Unemployment and ill health understanding the relationship. - In: Journal of Epidemiology and Community Health 48: 333-337.

Blasius, J. (2001): Korrespondenzanalyse. - München, Wien: R. Oldenbourg Verlag.

Bopp, M. \& F. Gutzwiller (1999): Entwicklung der Mortalität in der Schweiz seit 1950. II. Regionale Unterschiede innerhalb der Schweiz. - In: Schweizerische Medizinische Wochenschrift 129: 799-809.

Calmonte, R., Spuhler, T. \& W. Weiss (Koord.) (2000): Schweizerische Gesundheitsbefragung: Gesundheit und Gesundheitsverhalten in der Schweiz 1997. Detailergebnisse der 2. Schweizerischen Gesundheitsbefragung 1997. - Neuchâtel: Bundesamt für Statistik.

Joye, D., Schuler, M., Nef, R. \& M. Bassand (1988): Typologie der Gemeinden der Schweiz. Ein systemati- 
scher Ansatz nach dem Zentren-Peripherien-Modell. - Bern: Bundesamt für Statistik.

Koller, C. (2000): Regionale Unterschiede und Gesundheit. - In: Calmonte, R., Spuhler, T. \& W. WEISS (Koord.): Schweizerische Gesundheitsbefragung: Gesundheit und Gesundheitsverhalten in der Schweiz 1997. Detailergebnisse der 2. Schweizerischen Gesundheitsbefragung 1997. - Neuchâtel: Bundesamt für Statistik.

LoCHNER, K., KaWAChI, I. \& B.P. Kennedy (1999): Social capital: a guide to its measurement. - In: Health \& Place 5: 259-270.

Miles, A. (1991): Women, Health and Medicine. Milton Keynes, Philadelphia: Open University Press.

Mitchell, R., Gleave, S., Bartley, M., Wiggins, D. \& H. Joshi (2000): Do attitude and area influence health? A multilevel approach to health inequalities? - In: Health \& Place 6: 67-79.

Schuler, M. \& D. Joye (1997): Die Raumgliederung der Schweiz. - Bern: Bundesamt für Statistik.

Subramanian, S.V., Kawachi, I. \& B.P. Kennedy (2001): Does the state you live in make a difference? Multilevel analysis of self-rated health in the US. - In: Social Science \& Medicine 53: 9-19.

SundQuist, J. \& S.-E. Johannson (1997): Self-reported poor health and low educational level predictors for mortality: A population-based follow-up study of 39,156 people in Sweden. - In: Journal of Epidemiology and Community Health 51:35-40.

Wilkinson, R. (1996): Unhealthy Societies. The Afflictions of Inequality. - London and New York: Routledge.

\section{Zusammenfassung: Regionale Muster im gesund- heitlichen Wohlbefinden der Schweizer Bevölkerung. Untersuchung des gesundheitlichen Wohlbefindens aufgrund der Schweizerischen Gesundheitsbefragung 1997 und der Schweizer Raumtypologien}

Seit den 1990er Jahren werden für das gesundheitliche Wohlbefinden neben individuellen Verhaltensfaktoren oder dem sozioökonomischen Status auch kollektive, regionengebundene Charakteristiken wie der Wohnort und Nachbarschaften diskutiert. In dieser Studie wurden als kollektive Einflussfaktoren die 22 Gemeindetypologien nach Joye, Schuler, Nef und BASSAND bezüglich des gesundheitlichen Wohlbefindens unter Berücksichtigung von Alter, Geschlecht, Bildung und sozioprofessionellen Kategorien untersucht. Besonders das Alter, aber auch der sozioökonomische Status scheint ein stärkerer Prädiktor zu sein als die Raumtypen. In den Zentren und der Peripherie ist das gesundheitliche Wohlbefinden im Vergleich zum Landesmittel häufiger weniger gut. In den periurbanen und reichen sowie in den touristischen und semitouristischen Gemeinden fühlt man sich gesundheitlich «gut» bis «sehr gut».
Summary: Regional patterns in the state of health of the Swiss population. Investigation of state of health combining results from the Swiss Survey of Health in 1997 and Swiss spatial typologies

Since the 1990s, discussions on state of health have taken collective and regional characteristics such as place of residence or neighbourhoods into consideration, besides typical factors such as individual behaviour or socio-economic status. In this article, 22 community typologies as defined by Joye, Schuler, NeF and BASSAND were investigated with regard to states of health taking collective factors of influence into consideration, namely age, gender, level of education and socio-professional influences. In particular, the factor «age» appears to have a stronger influence on state of health results than spatial factors, although socio-economic status may also be considered an important factor. In city centres and in the rural periphery, personal state of health is often below the national average. In peri-urban and well-off areas, including touristic and semi-touristic destinations, people consider their state of health to be «good» to «very good».

Résumé: Modèles régionaux en matière de bien-être physique de la population suisse. Analyse du bienêtre physique sur la base de l'enquête suisse sur la santé, en 1997, et selon les typologies spatiales suisses Depuis les années 1990, la discussion est ouverte sur les rapports entre le bien-être physique, les facteurs de comportement individuels, le statut socio-économique, ainsi que les caractéristiques collectives régionales, telles que le lieu d'habitation et les voisinages. La présente étude comporte l'analyse des facteurs d'influence collectifs des 22 typologies de communes, selon Joye, Schuler, Nef et Bassand, en ce qui concerne le bien-être physique eu égard à l'âge, mais aussi au statut socio-économique, qui semble être un facteur d'influence plus important que les types spatiaux. Dans les centres et à la périphérie le bien-être physique est moins prononcé qu'en moyenne à la campagne. Dans les communes périurbaines et les communes touristiques et semi-touristiques riches, on se sent physiquement en «bonne» ou en «très bonne» forme.

Dr. Charis Keller-Lengen, Geographisches Institut, Universität Zürich, Winterthurerstrasse 190, CH-8057 Zürich, Schweiz.

e-mail:chkeller@geo.unizh.ch

\section{Manuskripteingang/received/manuscrit entré le} 5.4.2004

Annahme zum Druck/accepted for publication/accepté pour l'impression: 14.6.2005 